\title{
El proceso de planificación previa en la escritura colaborativa de textos en educación primaria
}

\section{The Process of Pre-Planning in Collaborative Writing at the Primary Level}

\section{o processo de planejamento prévio na escrita colaborativa de textos no ensino fundamental}

\author{
Mónica Aznárez-Mauleón* \\ *Universidad Pública de Navarra, Navarra / Espanha \\ monica.aznarez@unavarra.es \\ http://orcid.org/0000-0002-9363-8446 \\ Maite López-Flamarique** \\ **Universidad Pública de Navarra, Navarra / Espanha \\ maite.lopez@unavarra.es \\ http:/ / orcid.org/0000-0002-1980-6368
}

\begin{abstract}
RESUMEN: Este trabajo, de carácter descriptivo y cualitativo, investiga las operaciones o subprocesos de planificación textual que lleva a cabo el alumnado de primaria así como las técnicas que emplea en la elaboración de borradores. La investigación se basa en los modelos cognitivos de la escritura y en los principios de la teoría sociocultural del aprendizaje. Para llevar a cabo el estudio, se analizan los episodios encontrados en las interacciones orales de 11 parejas de $5^{\circ}$ de primaria de un centro de la región de Navarra (España) y los borradores que estas elaboran en el contexto de una tarea de escritura conjunta de textos expositivo-argumentativos. Los resultados muestran que, en general, el alumnado atiende principalmente al subproceso de generación de ideas, y, en menor medida, a aspectos metalingüísticos, estéticos y de gestión de la tarea. Respecto a las técnicas, se observa que solo seis parejas las utilizan y que presentan diferente nivel de complejidad. Estos resultados, junto a la gran variabilidad encontrada, evidencian la necesidad de trabajar este proceso de manera específica y sistemática en esta etapa.
\end{abstract}

PALABRAS CLAVE: planificación; escritura colaborativa; episodios; borradores; educación primaria. 


\begin{abstract}
This paper, of descriptive and qualitative nature, studies the operations and sub-processes of textual planning performed by primary students as well as the techniques they use when writing their drafts. To this end, we analyze the episodes found in the oral interactions of 11 pairs of $5^{\text {th }}$ graders and the drafts they produced in the context of a joint expository-argumentative writing task. Results show that, in general, students focus on the sub-process of the generation of ideas, and, to a lesser extent, on metalinguistic, esthetic and task management aspects. Regarding the techniques, we observe that only six pairs use them and that they represent different levels of complexity. These results, together with the high variability found, evidence the need to practice this process specifically and systematically at this stage.
\end{abstract}

KEYWORDS: planning; collaborative writing; episodes; drafts; primary education.

RESUMO: Este trabalho, de caráter descritivo e qualitativo, investiga as operações ou subprocessos de planejamento textual realizados por alunos do ensino fundamental, bem como as técnicas utilizadas na elaboração de rascunhos. A pesquisa é baseada nos modelos cognitivos de escrita e nos princípios da teoria sociocultural da aprendizagem. Para realizar o estudo, analisam-se os episódios encontrados nas interações orais entre 11 duplas de $5^{a}$ série primária de um centro da região de Navarra (Espanha) e os rascunhos que elas preparam no contexto de uma tarefa de escrita conjunta de textos expositivo-argumentativos. Os resultados mostram que, em geral, os alunos atendem principalmente ao subprocesso de geração de ideias e, em menor escala, aos aspectos metalinguísticos, estéticos e de gerenciamento de tarefas. Em relação às técnicas, observa-se que apenas seis duplas as utilizam e que apresentam diferentes níveis de complexidade. Estes resultados, juntamente com a grande variabilidade encontrada, demonstram a necessidade de trabalhar este processo de maneira específica e sistemática nesta etapa.

PALAVRAS-CHAVE: planejamento, redação colaborativa, episódios, rascunhos, ensino fundamental.

\title{
1 Introducción
}

La escritura es una competencia esencial no solo para la comunicación social sino para el propio aprendizaje, por lo que constituye, junto con la comprensión lectora, una de las prioridades de la educación primaria. El interés por descubrir cómo se desarrolla esta competencia y cómo llevan a cabo este proceso las personas expertas y las (todavía) inexpertas como el alumnado de primaria, ha motivado un gran número de investigaciones en las últimas décadas. Algunas de ellas han destacado el importante papel que desempeña el proceso de planificación previa en la mejora de la escritura (LIMPO; ALVES; FIDALGO, 2014), por lo que analizar cómo lleva a cabo 
este proceso el alumnado con el fin de orientar su enseñanza-aprendizaje resulta especialmente relevante.

Gran parte de los trabajos que han estudiado esta cuestión se han basado en el análisis de los llamados "protocolos individuales de pensamiento en voz alta" (think-aloudprotocols) (BEREITER; SCARDAMALIA, 1987), en el análisis de los borradores y de los textos producidos por el alumnado (LIMPO; ALVES; FIDALGO, 2014), o en la entrevista cognitiva (ARROYO; SALVADOR, 2005; GALLEGO ORTEGA, 2008). Sin embargo, apenas hemos encontrado estudios que analicen el proceso de planificación previa en tareas de escritura conjunta de textos. El análisis de las interacciones orales en este tipo de tareas constituye una fuente de información muy valiosa que viene a complementar la obtenida mediante los métodos citados, pues permite descubrir la manera en la que se lleva a cabo el proceso de escritura mediante la observación de comportamientos más espontáneos que los protocolos (DAIUTE, 1986). Por otra parte, desde una perspectiva sociocultural (VYGOTSKY, 1986), es posible establecer la hipótesis de que los procesos observados en la escritura colaborativa serán los que, con el tiempo, el alumnado realizará de manera autónoma en la escritura individual.

El presente trabajo, de carácter descriptivo y cualitativo, pretende avanzar en el conocimiento de cómo lleva a cabo el proceso de planificación previa el alumnado de $5^{\circ}$ primaria a través, por un lado, del estudio de las interacciones que tienen lugar en una tarea de escritura en parejas de textos expositivo-argumentativos en español L1, y, por otro, del análisis de los borradores que elaboran. Concretamente, se quiere descubrir: (1) qué operaciones o subprocesos realiza el alumnado cuando se le pide que planifique un texto expositivo-argumentativo; (2) si este emplea o no técnicas de planificación en la elaboración de borradores; y, si es así; (3) de qué técnicas se trata.

El artículo se ha estructurado en siete secciones. La segunda sección constituye el marco teórico. La tercera explica la metodología del estudio: contexto y participantes, instrumentos y diseño, y análisis. En la cuarta se presentan los resultados en dos subsecciones: la correspondiente al análisis de las interacciones orales y la correspondiente al análisis de los borradores. La quinta y la sexta se corresponden con la discusión y con las implicaciones didácticas respectivamente, y en la séptima se presentan las conclusiones. 


\section{La planificación textual en la escritura infantil}

Tal como han mostrado diferentes modelos cognitivos de la escritura (BEREITER; SCARDAMALIA, 1987; FLOWER; HAYES, 1981), la planificación es, junto a la textualización y la revisión, uno de los procesos básicos en la producción textual. Las operaciones fundamentales que implica este proceso son la generación de ideas para el texto -que implica recuperar información de la memoria a largo plazo-, la organización de dichas ideas en una estructura coherente, y el establecimiento de objetivos -basado en aspectos como la intención del texto y los destinatarios a los que se dirige (FLOWER; HAYES, 1981).

Si bien todas las personas que escriben planifican de algún modo, los estudios han demostrado que el tipo de planificación que realizan las personas inexpertas difiere del que llevan a cabo las expertas. Así, en las investigaciones sobre la escritura infantil se ha observado que, mientras planifican, los/as niños/as se centran en la generación de ideas (BEREITER; SCARDAMALIA, 1987; LANGER, 1986; MCCUTCHEN, 1988) y no suelen establecer objetivos de escritura ni utilizar conocimientos sobre la estructura textual para organizar el contenido (MACARTHUR; HARRIS; GRAHAM, 1994), si bien son capaces de llevar a cabo estas operaciones, como se ha comprobado en tareas significativas de escritura (LEE; KARMILOFF-SMITH; CAMERON; DODSWORTH, 1998; LITTLETON, 1998). Los resultados obtenidos en el contexto español por Arroyo y Salvador (2005) parecen apuntar en la misma línea, ya que la organización de ideas es la operación de planificación que el alumnado dice tener menos en cuenta.

Por otro lado, con respecto al momento en el que se lleva a cabo este proceso, se ha comprobado que la planificación textual puede realizarse tanto antes de escribir como mientras se escribe (MATSUHASHI, 1981; SCHILPEROORD, 2001). Sin embargo, la planificación "a tiempo real" no parece ser la mejor estrategia para las personas inexpertas, pues esta implica una sobrecarga para la memoria de trabajo, que tiene que atender simultáneamente a la planificación y a la textualización. Diferentes estudios han observado que, si bien el alumnado presenta esta habilidad ya en primaria, este tiende a planificar mientras escribe (SÉNÉCHAL; HILL; MALETTE, 2018), y solo conforme avanza en edad y con la práctica comienza a distinguir entre planificación y producción textual (BERNINGER; SWANSON, 1994; LIMPO; ALVES; FIDALGO, 2014). 
En este sentido, se ha observado que el alumnado más joven tiene dificultades para separar la planificación de la textualización, y que este planifica "generando texto" (BURTIS; BEREITER; SCARDAMALIA; TETROE, 1983). Concretamente, Bereiter y Scardamalia (1987) observaron que los/as niños/as de 10 años, a diferencia de otros/as de mayor edad (12-14 años), producían directamente una primera versión del texto cuando se les pedía que escribieran notas antes de empezar a escribir. Whitaker, Berninger, Johnston y Swanson (1994), en su estudio con alumnado de $4^{\circ}$ a $6^{\circ}$ de primaria, concluyeron que la planificación es un proceso cognitivo emergente que no está plenamente desarrollado o plenamente relacionado con el proceso de textualización en estas edades, en las que el alumnado realiza tanto planificación previa como concurrente. A este respecto, Limpo, Alves y Fidalgo (2014) señalan que la dificultad que presenta la planificación para el alumnado de primaria no es solo una cuestión de maduración sino que puede deberse al tipo de instrucción que este ha recibido.

En cuanto a los procedimientos de planificación empleados por el alumnado de primaria, se ha observado que estos presentan distintos grados de complejidad. Entre el alumnado de $4^{\circ}$ a $6^{\circ}$ que realizó planificación previa, Whitaker, Berninger, Johnston y Swanson (1994) identificaron como procedimientos, de más básicos a más complejos, las listas de proposiciones o palabras concretas (sin temas abstractos), las listas de temas sin subtemas, los temas con ejemplos y subtemas sin especificación de la relación estructural, y los esquemas o mapas de temas con ejemplos y con especificación de las relaciones estructurales. Como advierten Limpo, Alves y Fidalgo (2014) en su investigación con alumnado portugués de $4^{\circ}$ a $9^{\circ}$ grado (9 a 15 años), se puede hablar de una evolución en el tipo de técnicas empleadas según la edad. Así, las consideradas más básicas como las listas sin temas abstractos serían empleadas principalmente por el alumnado más joven, mientras las más elaboradas o complejas que reflejan relaciones estructurales (mediante, por ejemplo, elementos gráficos) serían más utilizadas por el alumnado de mayor edad. Finalmente, en el contexto español, entre los/as estudiantes de primaria entrevistados/as por Arroyo y Salvador (2005), algunos/as afirman que apuntan las ideas, otros/as que realizan "esquemas" o "resúmenes", y otros/as reconocen que no utilizan ningún procedimiento de registro de ideas porque ya las recuerdan cuando van a escribir.

Finalmente, existen numerosos estudios que han demostrado que la escritura del alumnado de primaria, tanto en inglés L1 (véase el meta- 
análisis de Graham, McKeown, Kiuhara y Harris, 2012) como en español L1 (LÓPEZ; TORRANCE; RIJLAARSDAM; FIDALGO, 2017; ROLDÁNPREGO; GONZÁLEZ-SEIJAS, 2016), mejora tras intervenciones didácticas centradas en las operaciones y en los procedimientos de planificación previa. El éxito de este tipo de intervenciones puede deberse a que la planificación permite al alumnado registrar las ideas que necesitará durante la escritura del texto y reduce la carga cognitiva que supone planificar durante la textualización (KELLOGG, 1988).

\section{Metodología}

El marco general en el que se basa este trabajo es el de la investigación cualitativa (TAYLOR; BOGDAN, 1987), ampliamente utilizada en el ámbito educativo como un enfoque adecuado para comprender lo que sucede en el aula (BOGDAN; BIKLEN, 2007). La presente investigación constituye un estudio de casos en el contexto de una tarea de producción en parejas de un texto expositivo-argumentativo que pretende descubrir: (1) a qué operaciones o subprocesos dedica el alumnado el tiempo de planificación previa a la escritura; (2) si este emplea o no procedimientos o técnicas de planificación en la elaboración de borradores; y, si es así, (3) de qué técnicas se trata. Esta investigación cualitativa se apoya asimismo en el análisis de la frecuencia con la que el alumnado, a lo largo de la interacción previa a la escritura final, realiza las diferentes operaciones.

\subsection{Contexto y participantes}

Este trabajo se ha realizado en un grupo-clase de 22 estudiantes (12 niños y 10 niñas) de $5^{\circ}$ de primaria (10-11 años) escolarizados en español en un centro concertado de la capital de Navarra (España) y con un nivel similar en la asignatura de lengua. Se eligió dicho curso porque a esta edad el alumnado, en general, ya ha automatizado el uso del código gráfico, lo que le permite dirigir la atención y el esfuerzo hacia procesos de orden superior (FLOWER; HAYES, 1981).

La sesión en la que se llevó a cabo la toma de datos pertenece a la asignatura de lengua castellana, la cual ocupa seis de las 28 sesiones semanales de 45 minutos que recibe el alumnado en el centro. Por último, el alumnado trabajó en parejas establecidas de modo aleatorio. 


\subsection{Diseño e instrumentos}

Para llevar a cabo la investigación se diseñó una tarea significativa de escritura (véase IMAGEN 1) para cuya realización el alumnado dispuso de 25 minutos. Junto con la consigna, se les entregó una hoja para la realización del borrador. Se decidió proponer la escritura de un texto expositivoargumentativo sobre el trabajo en equipo en la escuela, de modo que todo el alumnado pudiera generar contenido por tratarse de un tema cercano a su experiencia. Se prefirió este tipo textual porque, a pesar de formar también parte del currículo de primaria, este ha sido menos utilizado en las investigaciones que el narrativo.

IMAGEN 1 - Consigna de la tarea de escritura propuesta

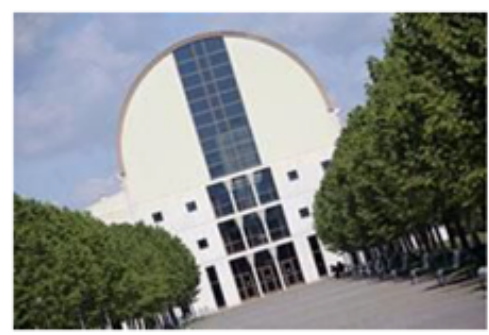

Un grupo de investigación de la Universidad Pública de Navarra está estudiando cuáles son los beneficios del trabajo en grupo en el aula y, entre sus intereses, desea conocer cuál es la opinión del alumnado con respecto a este tema.

Por eso, os pedimos que escribáis en parejas un texto en el que expliquéis qué tipo de trabajos en grupo hacéis en clase, cómo se llevan a cabo estos trabajos, cuáles son las ventajas e inconvenientes que creéis que tiene trabajar en grupo y si recomendariais esta forma de trabajo.

Antes de escribir, pensad sobre lo que vais a decir en el texto. Podéis usar este papel para hacer un esquema previo, pero escribid vuestro texto definitivo en la otra hoja. Por favor, cuidad la redacción y la limpieza. Tenéis hasta el final de esta sesión para realizar el trabajo.

iMuchas gracias por vuestra ayuda!

Para descubrir las operaciones que realizaba el alumnado durante el tiempo de planificación previa de la tarea, se utilizaron grabadoras de audio que registraron las interacciones orales de las 11 parejas participantes. Las grabaciones recogidas se analizaron posteriormente mediante el software de análisis cualitativo de datos Atlas.ti 8 (Scientific Software Development $\mathrm{GmbH}, 2017)$.

Por último, para conocer las técnicas de planificación previa empleadas por el alumnado, se analizaron los borradores producidos por cada pareja mediante los criterios de la Tabla 3. 


\subsection{Análisis}

Esta investigación implica el análisis de dos tipos de datos: las interacciones orales grabadas durante la tarea de escritura y los borradores elaborados por cada pareja. Las primeras se utilizan para responder a la primera pregunta de investigación: a qué operaciones dedica el alumnado la planificación previa. Los segundos sirven para responder a las otras dos preguntas de investigación, esto es, si el alumnado utiliza técnicas de planificación previa en sus borradores y, si es así, cuáles son. Si bien se trata de dos conjuntos de datos diferenciados, estos se han analizado de manera interrelacionada, ya que ofrecen información sobre el proceso y sobre el producto de una misma tarea de escritura.

$\mathrm{El}$ análisis de las interacciones orales se llevó a cabo directamente sobre los audios mediante el programa de análisis cualitativo Atlas.ti 8. Como advierten Markle, West y Rich, (2011) la codificación de datos multimedia, posible en la actualidad gracias a este tipo de programas, permite que el análisis sea eficaz, fiable y preciso.

La unidad de análisis que se ha adoptado en el estudio de las interacciones es el episodio. Esta unidad ha sido empleada en muchas investigaciones sobre la escritura conjunta de textos (STORCH, 2005, 2011; SWAIN; LAPKIN, 1995; CAMPS; GUASCH; MILIAN; RIBAS, 2000; GUTIÉRREZ, 2008; entre otros). La mayor parte de ellas se centran en los denominados language related episodes (LREs) que se definen como cualquier parte de diálogo en la que el alumnado habla sobre la lengua que está produciendo, cuestiona su uso lingüístico, o propone una corrección, tanto a su producción como a una ajena (SWAIN; LAPKIN, 1995). El presente trabajo no se centra en los LREs, sino que analiza cualquier tipo de episodio, esto es, cualquier fragmento de la interacción en la que el alumnado centra su atención en un aspecto del proceso de escritura. Así pues, los episodios pueden constar tanto de uno como de varios enunciados, ya que la delimitación se realiza con base en el contenido, es decir, se considera un nuevo episodio cada vez que la conversación cambia de foco de interés.

La segmentación y codificación de los datos orales se realizó en dos capas. En primer lugar, se segmentaron las interacciones por episodios y se identificaron aquellos en los que el alumnado explicitaba claramente un cambio de proceso como "A1: Vale, ahora empieza a escribir: En el colegio...”, “A1: Oye, ¿túuuu cuándo crees que podemos empezar a escribir? 
A2: Ahora", "A1: Yo lo iría pasando aquí porque quedan diez minutos", "A1: Venga, seguimos escribiendo, pero ahora en limpio"; es decir, episodios que marcan el final de la elaboración del borrador y el principio de la escritura del texto final. De este modo, y con el apoyo de lo escrito por cada pareja en el borrador y en el texto final, se delimitaron las fases de la tarea en las que se encontraba cada pareja a lo largo de la interacción (elaboración del borrador, escritura del texto final o revisión).

En segundo lugar, se procedió a codificar los episodios encontrados. A pesar de que el estudio se centra únicamente en la primera fase de la tarea (escritura del borrador), se segmentaron y codificaron las interacciones completas de modo que se pudiera contar con todo el contexto a la hora de interpretar los datos. La codificación de los episodios fue fundamentalmente deductiva, partiendo de las categorías correspondientes a las operaciones propias de la planificación textual, (generación de ideas, establecimiento de objetivos y organización textual) aunque se utilizó también el procedimiento inductivo para la codificación de los episodios relacionados con la escritura que no se correspondían con dichas categorías (episodios centrados en la gestión de la tarea y episodios dedicados a aspectos asociados a la textualización como los metalingüísticos - de puntuación, ortográficos, léxicos, morfosintácticos o textuales- o los estéticos). En las Tablas 1 y 2 se muestran los códigos utilizados a lo largo del estudio junto a ejemplos ${ }^{1}$ de episodios categorizados mediante cada uno de ellos. El análisis de las interacciones orales fue realizado por las dos investigadoras, tras la codificación conjunta de la interacción de una de las parejas y tras alcanzar un coeficiente alfa de acuerdo (KRIPPENDORFF, 1990) de 0.93 en la codificación de dos más de ellas.

\footnotetext{
${ }^{1}$ Los ejemplos se han transcrito utilizando las convenciones "A1/A2" (Alumno 1/Alumno 2), para indicar el miembro de la pareja que habla en cada momento; las mayúsculas (p. ej. “LEE”), para describir elementos contextuales importantes como que el/la alumno/a está leyendo en voz alta; y los corchetes para indicar solapamientos del discurso, es decir, palabras que se han emitido de manera simultánea. Se ha empleado "[XXX]" para indicar partes del discurso que resultan ininteligibles y "[...]” para fragmentos que omitimos por no ser relevantes.
} 
TABLA 1 - Operaciones de planificación. Códigos utilizados

y ejemplos de episodios

\begin{tabular}{|c|c|}
\hline Generación de ideas & $\begin{array}{l}\text { A1: ¿Qué tipo de trabajo hacéis en grupo hacéis en clase? } \\
\text { Pues hacemooos, aaaah... } \\
\text { A2: Ejercicios de mates } \\
\text { A1: Ejercicios de mates, sí. } \\
\text { (Pareja 1) }\end{array}$ \\
\hline $\begin{array}{l}\text { Establecimiento de } \\
\text { objetivos }\end{array}$ & $\begin{array}{l}\text { A1: Han dicho que lo negro era un poco lo que queríaaan... } \\
\text { es lo que está másss subrayao } \\
\text { A2: Sí sí lo que más importa ¿¿no? queee digamos. } \\
\text { A1: Sí (LEE LA CONSIGNA) } \\
\text { (Pareja 2) }\end{array}$ \\
\hline Estructura textual & $\begin{array}{l}\text { A1: Pero vamos a poner distin... ponemos distintas } \\
\text { asignaturas y entonces ahí ponemos lo que hacemos, lo que } \\
\text { ehmmm trabajamos en grupo, cómo lo hacemos, y eso... } \\
\text { (Pareja 10) }\end{array}$ \\
\hline $\begin{array}{l}\text { Conciencia sobre el } \\
\text { proceso de planificación }\end{array}$ & $\begin{array}{l}\text { A2: Ten en cuenta que esto es en sucio, no hay que poner } \\
\text { todo, hay que ponerlo aquí. } \\
\text { (Pareja 11) }\end{array}$ \\
\hline
\end{tabular}

TABLA 2 - Otras operaciones. Códigos utilizados y ejemplos de episodios

\begin{tabular}{|c|c|c|}
\hline \multirow{3}{*}{ Gestión de la tarea } & Gestión de roles & $\begin{array}{l}\text { A1: ¿Me ayudas un poco a hacer el } \\
\text { esquema, [tíooo]? } \\
\text { A2: [Vale], sí, sí... } \\
\text { (Pareja 8) }\end{array}$ \\
\hline & $\begin{array}{l}\text { Gestión del espacio } \\
\text { de escritura }\end{array}$ & $\begin{array}{l}\text { A1: Hazlo bastante junto pa que nos } \\
\text { quepa más. } \\
\text { (Pareja 5) }\end{array}$ \\
\hline & Gestión del tiempo & $\begin{array}{l}\text { A1: Vale cuatro minutos, repito dos } \\
\text { minutos pa cadaaa cosa } \\
\text { (Pareja 3) }\end{array}$ \\
\hline \multirow[t]{2}{*}{$\begin{array}{l}\text { Aspectos relacionados } \\
\text { con la textualización }\end{array}$} & $\begin{array}{l}\text { Aspectos } \\
\text { metalingüísticos }\end{array}$ & $\begin{array}{l}\text { A1: Cuando nos portamosss, regular } \\
\text { (RÍE), o cuando nos portamos mal, } \\
\text { ahí, todo directo. } \\
\text { A2: Buaah que noo } \\
\text { (Pareja 7) }\end{array}$ \\
\hline & Aspectos estéticos & $\begin{array}{l}\text { A1: ¿No puedes escribir una letra } \\
\text { más grande, por favor? } \\
\text { (Pareja 3) }\end{array}$ \\
\hline
\end{tabular}


En cuanto a los borradores, tras la comparación de todos ellos y con base en los procedimientos de planificación identificados por Whitaker, Berninger, Johnston y Swanson, (1994) se estableció una clasificación en cuatro niveles, desde la ausencia de técnicas de planificación hasta la elaboración de un esquema o mapa conceptual (véase TABLA 3).

\section{TABLA 3 - Clasificación de las técnicas utilizadas en la elaboración de borradores}

\begin{tabular}{ll}
\hline \multicolumn{1}{c}{ Técnica de planificación } & \multicolumn{1}{c}{ Descripción } \\
\hline No utiliza & Escribe el texto completo directamente \\
Ideas no organizadas & Escribe ideas sin ninguna organización \\
Ideas con organización emergente & $\begin{array}{l}\text { Escribe temas que ejemplifica con al menos una } \\
\text { idea. No establece relación entre los temas }\end{array}$ \\
Esquema o mapa conceptual & $\begin{array}{l}\text { Al menos dos temas con ejemplos concretos. Se } \\
\text { establece relación estructural entre los temas }\end{array}$ \\
\hline
\end{tabular}

\section{Resultados}

\subsection{Resultados del análisis de las interacciones}

El análisis de los 200 episodios registrados durante la planificación previa ha revelado que el alumnado se centra en muy diversos aspectos, desde aquellos que se consideran propios de la planificación textual como tal (generación de ideas, establecimiento de objetivos y estructura textual) o los estrechamente relacionados con ella (manifestación de la conciencia sobre este proceso), hasta los que tienen que ver con la gestión de la tarea (gestión de roles, gestión del espacio de escritura y gestión del tiempo) y los que se pueden asociar a la textualización (aspectos metalingüísticos y estéticos). En la Tabla 4 se muestra, de mayor a menor, el número de episodios que dedica el alumnado durante la elaboración del borrador a los diferentes aspectos, y en la Tabla 5, los porcentajes de episodios agrupados según su naturaleza (planificación textual, gestión de la tarea y aspectos asociados a la textualización). 
TABLA 4 - Número de episodios dedicados a cada aspecto en la planificación previa

\begin{tabular}{|c|c|c|c|c|c|c|c|c|c|c|}
\hline & 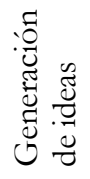 & $\frac{\mathscr{U}}{\mathscr{O}}$ & 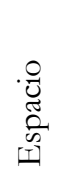 & $\frac{a}{0}$ & 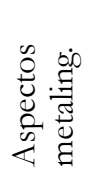 & 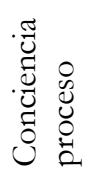 & 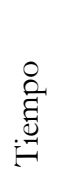 & 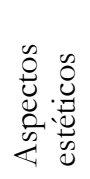 & 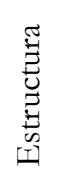 & 胥 \\
\hline P1 & 7 & 4 & 5 & 2 & 0 & 1 & 0 & 1 & 0 & 20 \\
\hline P2 & 5 & 6 & 2 & 4 & 0 & 0 & 0 & 1 & 2 & 20 \\
\hline P3 & 0 & 3 & 7 & 4 & 0 & 1 & 3 & 1 & 0 & 19 \\
\hline P4 & 7 & 1 & 3 & 3 & 0 & 3 & 2 & 2 & 0 & 21 \\
\hline P5 & 2 & 2 & 0 & 1 & 4 & 3 & 0 & 1 & 0 & 13 \\
\hline P6 & 10 & 1 & 0 & 0 & 2 & 0 & 0 & 1 & 0 & 14 \\
\hline P7 & 4 & 2 & 0 & 3 & 3 & 1 & 1 & 2 & 0 & 16 \\
\hline P8 & 8 & 1 & 0 & 1 & 0 & 2 & 1 & 1 & 0 & 14 \\
\hline P9 & 3 & 1 & 0 & 1 & 0 & 0 & 0 & 1 & 0 & 6 \\
\hline P10 & 12 & 3 & 5 & 1 & 4 & 2 & 1 & 0 & 3 & 31 \\
\hline P11 & 10 & 5 & 1 & 2 & 2 & 1 & 4 & 0 & 1 & 26 \\
\hline Total & 68 & 29 & 23 & 23 & 15 & 14 & 12 & 10 & 6 & 200 \\
\hline
\end{tabular}

TABLA 5 - Porcentajes de episodios de planificación previa agrupados según su naturaleza

\begin{tabular}{|c|c|c|c|c|c|c|c|c|c|}
\hline & \multicolumn{4}{|c|}{ Planificación textual } & \multicolumn{3}{|c|}{ Gestión de la tarea } & \multicolumn{2}{|c|}{$\begin{array}{c}\text { Aspectos } \\
\text { asociados a la } \\
\text { textualización }\end{array}$} \\
\hline & 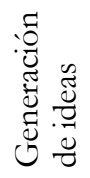 & $\begin{array}{l}\infty \\
0 \\
. \frac{0}{0} \\
\frac{0}{0}\end{array}$ & 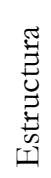 & 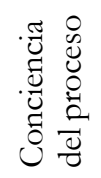 & $\frac{\mathscr{e}}{\mathscr{O}}$ & $\begin{array}{l}0 \\
\frac{0}{U} \\
\frac{\tilde{O}}{0} \\
\text { II }\end{array}$ & 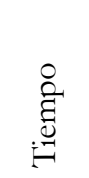 & 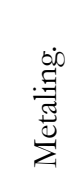 & 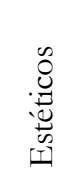 \\
\hline P1 & 35 & 10 & 0 & 5 & 20 & 25 & 0 & 0 & 5 \\
\hline P2 & 25 & 20 & 10 & 0 & 30 & 10 & 0 & 0 & 5 \\
\hline P3 & 0 & 21,05 & 0 & 5,26 & 15,79 & 36,84 & 15,79 & 0 & 5,26 \\
\hline P4 & 33,33 & 14,29 & 0 & 14,29 & 4,76 & 14,29 & 9,52 & 0 & 9,52 \\
\hline
\end{tabular}




\begin{tabular}{cccccccccc} 
P5 & 15,38 & 7,69 & 0 & 23,08 & 15,38 & 0 & 0 & 30,77 & 7,69 \\
P6 & 71,43 & 0 & 0 & 0 & 7,14 & 0 & 0 & 14,29 & 7,14 \\
P7 & 25 & 18,75 & 0 & 6,25 & 12,50 & 0 & 6,25 & 18,75 & 12,50 \\
P8 & 57,14 & 7,14 & 0 & 14,29 & 7,14 & 0 & 7,14 & 0 & 7,14 \\
P9 & 50 & 16,67 & 0 & 0 & 16,67 & 0 & 0 & 0 & 16,67 \\
P10 & 38,71 & 3,23 & 9,68 & 6,45 & 9,68 & 16,13 & 3,23 & 12,90 & 0 \\
P11 & 38,46 & $\mathbf{7 , 6 9}$ & 3,85 & 3,85 & 19,23 & 3,85 & 15,38 & 7,69 & 0 \\
\hline Total & $\mathbf{3 4}$ & $\mathbf{1 1 , 5 0}$ & $\mathbf{3}$ & $\mathbf{7}$ & $\mathbf{1 4 , 5 0}$ & $\mathbf{1 1 , 5 0}$ & $\mathbf{6}$ & $\mathbf{7 , 5 0}$ & $\mathbf{5}$ \\
\hline
\end{tabular}

En términos globales, se puede afirmar que aproximadamente la mitad de los episodios se dedica a las operaciones propias de la planificación textual: un $34 \%$ a la generación de ideas, un 11,5\% al establecimiento de objetivos, y un $3 \%$ a la estructura. Como se vio en la Tabla 1 , se incluyen en este grupo los episodios en los que el alumnado manifiesta de algún modo su conciencia sobre el proceso de planificación, que en este caso representan un 7\% del total. Así pues, se puede afirmar que, en términos generales, el aspecto al que más atención presta el alumnado en la planificación previa es la generación de ideas, si bien encontramos que existe bastante variabilidad entre parejas: las parejas 6 y 8 dedican respectivamente el 71,43\% y el 57,14\% de los episodios de planificación a la generación de contenido, mientras las parejas 3 y 5 dedican menos de un 16\% a esta labor, pues su interacción se centra en otros aspectos como la gestión de la tarea en el caso de la pareja 3 y los aspectos metalingüísticos en el caso de la pareja 5. A continuación se ofrecen algunos ejemplos de los numerosos episodios en los que el alumnado genera ideas para el texto:

Ejemplo 1:

A1: El trabajar en grupo es...

A2: Más fácil, tienes más ideas...

A1: Sí, es más fácil yy te pueden ayudar a hacer bibliografías, jaja

A2: (ESCRIBE) Más...

(Pareja 4) 
Ejemplo 2:

A1: A mí me gusta trabajar en grupo pero depende en qué cosas o sea...

A2: Dimeee en qué te parece que es bueno, ¿no?

$[\ldots]$

A1: Por ejemplo a ver me parece bien trabajar en grupo porque a ver pues pooor porqueee imagínate que uno no sabe algo pues le podemos ayudar y eso

A2: Yaaa y enci[maaaa]

A1: [Yyy] ayudarle a hacer cosas y bueno en el grupo pues estamooos

A2: Yaa y y en grupo se puede trabajaaar mucho mejooor, porque es más fácil hacerlooo con más gente...

A1: Sí

A2: Que solo

(Pareja 8)

Se observa que el alumnado se apoya en la consigna tanto para la generación de contenido para el texto como para el establecimiento de objetivos. En los episodios dedicados a este último aspecto, el alumnado vuelve sobre la consigna para tener claro, principalmente, qué tiene que escribir:

Ejemplo 3:

A1: Hay que escribir un texto con las ventajas, eh deee, un texto... qué tipo de trabajos en grupo hacemoos, en clasee, cuáles son las ventajas, cuáles son los inconvenientes, y si recomendaríamos este trabajo.

(Pareja 5)

En cuanto a la organización o estructura textual, son solo tres las parejas que tratan este aspecto, y lo hacen, además, en muy pocos episodios. En la mayor parte de ellos, estas parejas relacionan la estructura textual con los objetivos de la tarea y con la distribución del texto en la página, esto es, con el espacio de escritura: 


\section{Ejemplo 4:}

A1: [...] qué tipo de trabajos en grupo hacéis en clase, cómo se llevan a cabo estos trabajos. Vamos a empezar primero eso, luego cuáles son las ventajas e incon[venientes]

A2: [S1]

A2: Yyy si lo [recomendaríamos]

A1: [Y después] si lo recomendaríamos. [Vale]

A2: [Yyy] ponemos la recomendación al final, o sea por ejemplo, mira, este es el párrafo yo qué sé, esto es el párrafo deee lo que escribimos en $[\mathrm{XXX}]$ lo que hacemos en claseee y cómo trabajamos lo dee, en grupo, esto así, (ESCRIBE) baa baa baa

$[\ldots]$

A1: Aquí podemos poner lo deee qué tipo de trabajos en grupo hacéis en clase, cómo se llevan a cabo y todo eso, y luego aquí lo eehh, aquí, ehh las ventajas e inconvenientes y aquí si lo recomendaríamos, ya está.

(Pareja 2)

Por último, se han encontrado algunos episodios en los que el alumnado muestra tener conciencia de lo que implica realizar una planificación previa. Mientras algunas parejas simplemente hacen referencia al hecho de "escribir en sucio" (parejas 5 y 7), otras hablan de realizar un esquema, de no escribirlo todo en el borrador y de ordenar lo que se quiere decir:

Ejemplo 5:

A1: Bueno, a ver, empezamoos aaa hacer un poco esquema, de lo que vamos a hacer aquí ¿no?

A2: Eeeh, sí o sea porque esto es lo queee, vale.

(Pareja 8)

Ejemplo 6:

A1: Pero yo creo que no tendríamos que empezar a escribirlo (SILENCIO). Porqueee, tenemos que ponerlo en orden, y no desordenao.

(Pareja 11) 
En cuanto a los episodios restantes, es decir, aquellos que hemos considerado que no están relacionados con la planificación del texto propiamente dicha, la mayor parte de ellos se dedica a cuestiones relacionadas con la gestión de la tarea: gestión de roles (ejemplo 7) y gestión del espacio de escritura y del tiempo (ejemplos 8 y 9).

Ejemplo 7:

A1: Mira yo voy escribiendo aquí como en el texto y tú vas poniendo aquí en plan opciones.

(Pareja 3)

Ejemplo 8:

A1: Bueno, ¿lo hacemos ya en la hoja sagrada?

(Pareja 1)

Ejemplo 9:

A1: Creo que deberíamos empezar a hacerlo aquí ¿eh? Es que no nos va a dar tiempo

A2: Vale pues vamos a ir haciéndolo ahí.

(Pareja 4)

Los episodios menos frecuentes son los dedicados a aspectos que se asocian a la textualización como los metalingüísticos y los estéticos. Los escasos episodios dedicados a aspectos metalingüísticos se refieren principalmente al léxico, a la ortografía y a la puntuación. A este respecto, debemos decir que, como se verá más adelante en el análisis de los borradores, y como cabría esperar, tres de las cinco parejas que realizan episodios metalingüísticos durante la planificación escribieron el texto completo en el borrador (parejas 5, 6 y 7), lo que explica que presten más atención que otras parejas a aspectos de este tipo como la ortografía y la puntuación (véase ejemplo 10). Sin embargo, los episodios de este tipo realizados por las parejas 10 y 11 muestran que estas, mientras realizan operaciones de planificación, también atienden a aspectos lingüísticos, en estos casos, especialmente, al léxico (véanse ejemplos 11 y 12). Se observa por tanto que, al tiempo que generan ideas y escriben en el borrador, estas parejas discuten ya el modo de textualizar dichas ideas, es decir, las palabras concretas que van a utilizar en el texto. 
Ejemplo 10:

A1: Pero, coma, no, ¿o iría aquí antes la coma? Porque, pero, sí

A2: Perooo

A1: Y aquí iría la coma

(Pareja 7)

Ejemplo 11:

A1: Aprendemos (ESCRIBE) Apreeen...

A2: Aprendemos nuevas cosas

A1: Cosas... Bueno aprendemooos, aprendemos eehh distintos temas (Pareja 10)

Ejemplo 12:

A1: Las ideas son más creativas

A2: Vale; nooo hay más ideas

A1: ¿Eh?

A2: Las ideas van a ser lo mismo de creativas pero va a haber más ideas $[\ldots]$

A1: Pero mira uno dice una idea entonces luego otro la mejora y asíii, acaban siendo creativas

A2: Creativo es hacer algo que no ha hecho nadie

A1: Bueno, oooo no, a ver

A2: Creativo es el que inventa la pregunta, no los que la mejoran.

(Pareja 11)

Finalmente, los aspectos estéticos de la escritura que trata el alumnado son la caligrafía (ejemplo 13) y la limpieza del escrito.

Ejemplo 13:

A1: Haz una buena letra, por favor.

(Pareja 10)

\subsection{Resultados del análisis de los borradores}

Como muestra la Tabla 6, el análisis de lo escrito por cada pareja en los borradores muestra que, de los cuatro niveles de uso de procedimientos 
de planificación que se han establecido, cinco de las 11 parejas (parejas 2, 5, 6,7 y 9) se encuentra en el más bajo, pues no utilizan ningún procedimiento de planificación sino que redactan en forma de texto las ideas que generan (véase IMAGEN 2); otras cinco se encuentran en los niveles medios, ya que utilizan técnicas sintéticas de complejidad baja o media (parejas 1, 3, 4 y 11); y tan solo dos (parejas 8 y 10) se encuentran en el nivel más alto, el correspondiente al uso de esquemas y mapas conceptuales. Es interesante señalar que la pareja 10 emplea dos procedimientos de planificación, pues elabora una lista de ideas que posteriormente organiza en un mapa conceptual a partir de los requerimientos de la consigna.

TABLA 6 - Uso de técnicas de planificación por parejas

\begin{tabular}{cccc}
\hline No utiliza & $\begin{array}{c}\text { Ideas no } \\
\text { organizadas }\end{array}$ & $\begin{array}{c}\text { Ideas con } \\
\text { organización } \\
\text { «emergente" }\end{array}$ & $\begin{array}{c}\text { Esquema o mapa } \\
\text { conceptual }\end{array}$ \\
\hline P1 & $\checkmark$ & $\checkmark$ & \\
\hline P2 & $\checkmark$ & $\checkmark$ & \\
\hline P4 & $\checkmark$ & & \\
\hline P5 & $\checkmark$ & & $\checkmark$ \\
\hline P6 & $\checkmark$ & & $\checkmark$ \\
\hline P7 & $\checkmark$ & & \\
\hline P8 & $\checkmark$ & & \\
\hline P9 & & & \\
\hline P10 & $\checkmark$ & & \\
\hline P11 & $\checkmark$ & & \\
\hline
\end{tabular}

Como hemos apuntado, las técnicas o procedimientos de planificación del contenido que utilizan seis de las 11 parejas implican procesos cognitivos de diferente nivel de complejidad: la técnica de anotar algunas de las ideas que aparecerán después en la producción final (pareja 3) -que sería la que se puede considerar más básica-; la de agrupar las ideas por temas -que requiere algo más de esfuerzo que la anterior (parejas 1, 4, 11) (véase IMAGEN 3)-; 
$y$, finalmente, la de elaborar un esquema o un mapa conceptual en el que, además de ejemplificar los temas, se refleja la relación que existe entre ellos mediante elementos gráficos como flechas o líneas (parejas 8 y 10) (véase IMAGEN 4). En concreto, la pareja 10 es la que utiliza la técnica cognitivamente más exigente. Estos procedimientos le sirven al alumnado fundamentalmente para registrar las ideas que genera y, en algunos casos (parejas 1, 4, 8, 10 y 11), también para organizarlas.

En los borradores, al igual que en los episodios, se observa que tanto las parejas que no utilizan ninguna técnica de planificación como las que sí lo hacen se basan en la consigna para generar el contenido, aunque no todas las parejas siguen el orden establecido en ella (descripción del tipo de trabajo que realizan en grupo en primer lugar, seguida de ventajas e inconvenientes y finalmente la recomendación).

IMAGEN 2 - Borrador de la pareja 5 (texto)

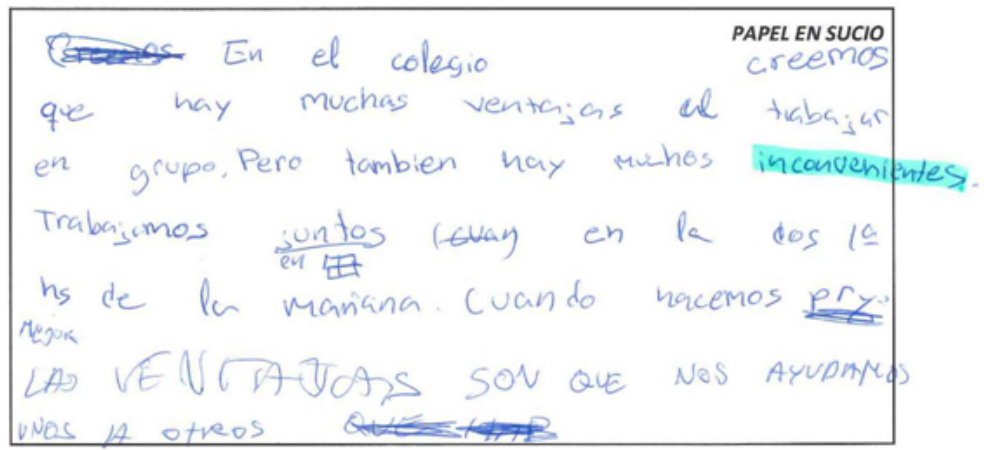

IMAGEN 3 - Borrador de la pareja 4 (lista de ideas organizadas) incomberientes

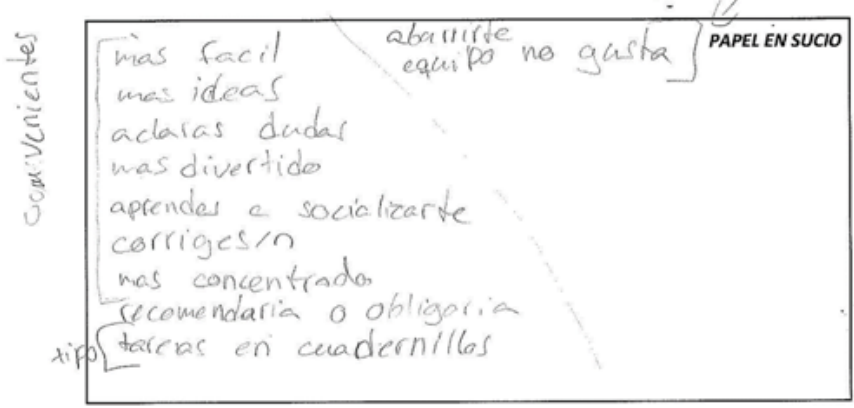


IMAGEN 4 - Borrador de la pareja 10 (mapa conceptual)

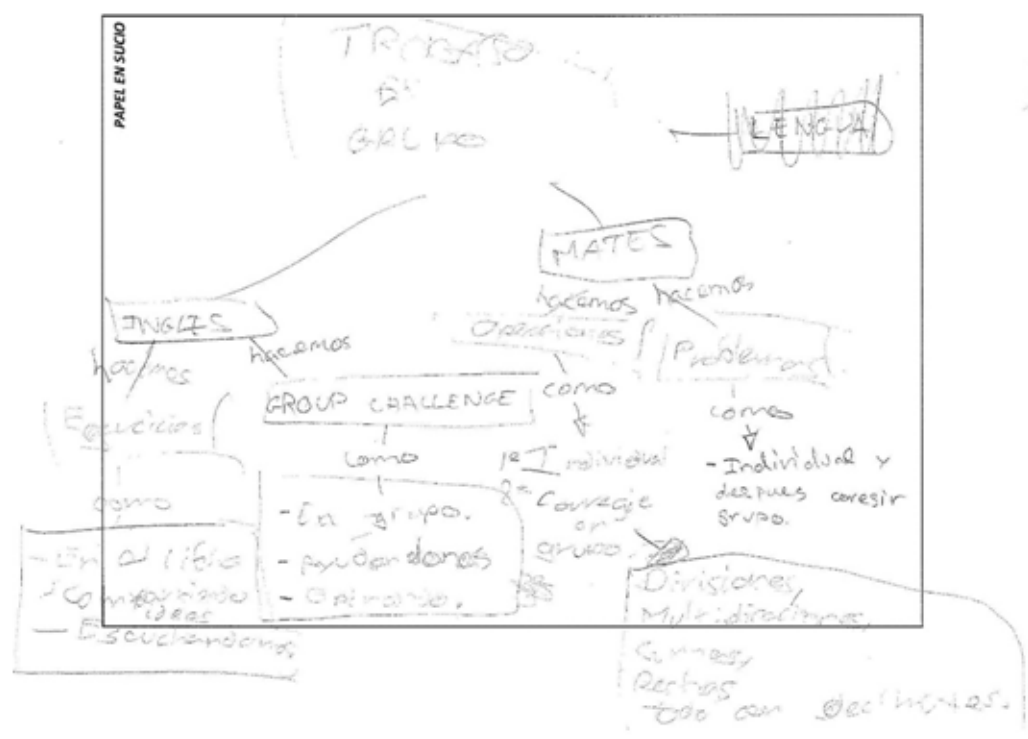

\section{Discusión}

El primer dato general que se debe destacar de nuestros resultados es la gran variabilidad que se observa entre las 11 parejas estudiadas, tanto en lo que respecta al contenido de sus interacciones durante la planificación previa como en lo relativo al uso de procedimientos de planificación en sus borradores. Esta variabilidad, que puede deberse a diferencias individuales como puede ser el hábito lector (SÉNÉCHAL; HILL; MALETTEE, 2018), se encuentra en línea con el carácter emergente de este proceso observado por Whitaker, Berninger, Johnston y Swanson (1994) en cursos medios de primaria, en los que una parte del alumnado lleva a cabo, con mayor o menor habilidad, el proceso cognitivo de planificación previa a la escritura, mientras otra parte no lo ha integrado todavía. Concretamente, nuestro análisis de los episodios y, especialmente, de los borradores ha revelado que parte del alumnado no distingue entre los procesos de planificación previa y de textualización en la escritura de textos expositivo-argumentativos, tal como se ha observado en la escritura individual de textos narrativos en estas edades (BERNINGER; SWANSON, 1994; LIMPO; ALVES; FIDALGO, 2014; SÉNÉCHAL; HILL; MALETTE, 2018). El hecho de que cinco de las 11 parejas escriban directamente el texto final en el borrador muestra, 
como observaron Bereiter y Scardamalia (1987), y Whitaker, Berninger, Johnston y Swanson (1994), que una parte del alumnado de estas edades no utiliza procedimientos de planificación y que planifica generando texto. La generación de texto durante la planificación previa explica también el hecho de que algunas de estas parejas dediquen parte de su interacción a hablar sobre aspectos metalingüísticos o estéticos, los cuales, si se quiere evitar la sobrecarga cognitiva (KELLOGG, 1988), sería más adecuado abordar durante la textualización.

En lo que respecta a los resultados relativos a la primera pregunta de investigación, el análisis de los episodios que tienen lugar en las interacciones ha mostrado que las operaciones a las que el alumnado dedica el tiempo de planificación previa no se corresponden en su totalidad con las operaciones consideradas propias de este proceso (generación de ideas, establecimiento de objetivos y organización textual). Así, hemos comprobado que, en general, el alumnado dedica alrededor de la mitad de los episodios a estas operaciones - principalmente a la primera -, mientras cerca de la otra mitad la dedica a la gestión de la tarea $(32 \%)$ y, en algunos casos, a aspectos metalingüísticos $(7,5 \%)$ y estéticos $(5 \%)$, los cuales sería más adecuado abordar durante la textualización. Entre los episodios dedicados a la gestión de la tarea, destacan aquellos en los que las parejas gestionan los roles que cada miembro va a asumir, lo cual es esencial en una tarea colaborativa como la propuesta. No sorprende tampoco, dadas las características de la tarea, que el alumnado preste atención en sus interacciones al espacio de escritura y al tiempo que tienen para realizarla.

En cuanto a la naturaleza de los episodios dedicados a las operaciones de planificación previa, nuestros datos concuerdan con los resultados obtenidos en los estudios realizados en inglés L1 (BEREITER; SCARDAMALIA, 1987; LANGER, 1986; MCCUTCHEN, 1988), ya que la mayor parte de los episodios realizados por el alumnado de nuestra muestra se dedica a la generación de ideas. Con respecto al subproceso de establecimiento de objetivos para el texto, en la línea de lo observado por Lee, Karmiloff-Smith, Cameron, y Dodsworth (1998) y Littleton (1998), se ha comprobado que el alumnado realiza esta operación en el contexto de la tarea significativa de escritura propuesta, ya que se han encontrado bastantes episodios en los que este vuelve sobre la consigna con el fin de tener claro sobre qué tiene que escribir. Finalmente, el escaso número de episodios dedicados a la estructura textual viene a confirmar los datos obtenidos 
por MacArthur, Harris y Graham (1994) y por Arroyo y Salvador (2005), quienes observan que la organización de las ideas parece ser una operación de planificación muy poco realizada por el alumnado de estas edades. Sin embargo, como comentaremos más adelante, nuestros datos también muestran que algunas parejas, aunque no hablen de ello en sus interacciones, sí organizan las ideas en sus borradores mediante procedimientos como la lista de ideas agrupadas por temas, el esquema o el mapa conceptual. En estos casos, la organización de ideas no se realiza de manera espontánea sino siguiendo - más o menos fielmente - el orden de las cuestiones que se plantean claramente en la consigna, lo cual puede explicar que no se genere ningún tipo de discusión al respecto en las interacciones.

En cuanto a los resultados relativos a nuestra segunda pregunta de investigación, el análisis de los borradores ha mostrado que solo seis de las 11 parejas utiliza algún procedimiento de planificación, mientras las cinco parejas restantes escriben directamente el texto. Es interesante señalar a este respecto que son estas cinco parejas las que parecen mostrar menos conciencia sobre el proceso de planificación en sus interacciones, pues solo dos de ellas (parejas 5 y 7) se refieren a él, identificándolo simplemente con "escribir en sucio".

Por último, en respuesta a la tercera pregunta de investigación, nuestros resultados muestran, en línea con lo observado por Whitaker, Berninger, Johnston y Swanson (1994) y Limpo, Alves y Fidalgo (2014), que los procedimientos de planificación empleados por las seis parejas que realizan una planificación previa en sus borradores son variados y de diferente nivel de complejidad, desde el registro de ideas hasta el esquema o mapa conceptual, aunque la mayoría utiliza procedimientos de nivel medio como la lista de ideas y la lista de ideas organizadas o jerarquizadas. Se ha de tener en cuenta, no obstante, que, al igual que en los citados trabajos, en la tarea propuesta en nuestro estudio se pedía al alumnado que planificara el texto antes de comenzar a escribirlo, y se le proporcionaba una hoja para ello. No podemos asumir, por tanto, que el alumnado hubiera hecho lo mismo motu proprio en ausencia de dicha petición.

\section{Implicaciones didácticas}

Como se ha visto, los resultados de esta investigación muestran diferentes comportamientos del alumnado cuando se le pide que realice una planificación previa en el contexto de una tarea de escritura colaborativa 
de un texto expositivo-argumentativo: una parte del alumnado escribe directamente el texto sin utilizar ningún procedimiento de planificación y otra parte utiliza procedimientos diversos con diferente nivel de exigencia cognitiva. Si bien esta heterogeneidad puede deberse al carácter emergente de la habilidad de planificación del alumnado de estas edades (WHITAKER; BERNINGER; JOHNSTON; SWANSON, 1994), también puede ser consecuencia de una instrucción insuficiente o exclusivamente centrada en el producto (LIMPO; ALVES; FIDALGO, 2014; TORRANCE; FIDALGO; GARCÍA, 2007). Así pues, y dado que esta habilidad no se desarrolla espontáneamente, sería recomendable trabajar, desde edades tempranas y de forma sistemática (ROLDÁN-PREGO; GONZÁLEZ-SEIJAS, 2016), la enseñanza y aprendizaje de técnicas que ayuden al alumnado a llevar a cabo el proceso de planificación de este tipo de textos, desde las más sencillas hasta las más complejas. Como hemos apuntado más arriba, la utilización de técnicas de planificación previa conlleva una descarga cognitiva que permite al alumnado dirigir su esfuerzo hacia los demás procesos de escritura (KELLOGG, 1988), lo que contribuye a la mejora de sus producciones escritas (GRAHAM; MCKEOWN; KIUHARA; HARRIS, 2012). Como advierten López, Torrance, Rijlaarsdam y Fidalgo (2017), tanto la instrucción directa como la observación y emulación de modelos de uso de estos procedimientos pueden resultar efectivas en estas edades. Este tipo de intervenciones didácticas ayudará al alumnado a centrarse en los aspectos que resultan relevantes para la planificación textual (generación de contenido, establecimiento de objetivos y organización de ideas) y a reducir la atención que presta a aspectos que resultaría más adecuado tratar durante la textualización como los metalingüísticos y estéticos. Además, como observa Villas Boas (2014), las técnicas de planificación abordadas en dichas intervenciones podrían ser transferidas a la escritura que el alumnado realice en cualquiera de las lenguas del currículo.

Por último, nuestro estudio también pone de manifiesto el importante papel que desempeña la consigna de la tarea a la hora de realizar la planificación del texto, pues esta ha servido de guía para elaborar los borradores, para la generación de ideas y para la organización de los textos expositivo-argumentativos. En este sentido, es importante que las consignas de las tareas de escritura que se plantee al alumnado de estas edades sean claras, ordenadas y completas, de modo que, con el tiempo y la práctica, y junto a intervenciones didácticas adecuadas como las arriba citadas, este vaya 
adquiriendo la habilidad de planificar sus textos de forma progresivamente más autónoma.

\section{Conclusiones}

El estudio de las interacciones orales de las 11 parejas de $5^{\circ}$ de primaria registradas durante la planificación previa a la escritura de un texto expositivo-argumentativo y el análisis de los borradores por ellas realizados ha permitido conocer las operaciones que estos/as estudiantes llevan a cabo durante este proceso así como las técnicas que emplean en la elaboración de sus borradores.

En primer lugar, en respuesta a nuestra primera pregunta de investigación, esto es, las operaciones que realiza el alumnado durante la planificación previa, el análisis de episodios ha mostrado que, en términos generales, el alumnado dedica aproximadamente la mitad de ellos a los subprocesos propios de la planificación, principalmente a la generación de ideas. El resto de episodios se dedican a aspectos que se pueden considerar relevantes antes de comenzar la escritura en este tipo de tarea - como el rol que asumirá cada miembro de la pareja -, pero también a cuestiones que se suelen asociar a la textualización como los aspectos metalingüísticos y estéticos de la escritura.

En segundo lugar, en relación a las otras dos preguntas de investigación, es decir, si el alumnado emplea técnicas de planificación en la elaboración de borradores y cuáles emplea, se observa una gran variedad de comportamientos: cinco de las 11 parejas escriben directamente el texto en el borrador, por lo que no emplean ningún procedimiento de planificación previa, mientras las seis parejas restantes utilizan técnicas de diferente nivel de complejidad, desde la lista de ideas no organizadas hasta el esquema o el mapa conceptual.

Nuestros resultados sugieren que, si bien el alumnado de $5^{\circ}$ de primaria es capaz de realizar una planificación previa para la escritura de un texto expositivo-argumentativo, una parte considerable de este tiene dificultades para distinguirla de la textualización, pues casi la mitad de las parejas planifican generando texto, no utilizan ninguna técnica de planificación y, algunas de ellas centran su atención en aspectos que sería más adecuado abordar durante la escritura. Por ello, es necesario trabajar este proceso sistemáticamente mediante, entre otros tipos de intervención, la 
práctica de tareas con consignas claras y completas como la utilizada en este trabajo, que sirvan de guía para realizar los subprocesos de la planificación que el alumnado deberá realizar de manera progresivamente más autónoma.

Somos conscientes, no obstante, de que sería necesario determinar hasta qué punto los resultados de este estudio se pueden extrapolar a la escritura individual. Asimismo, debemos reconocer que el tamaño de la muestra y el carácter descriptivo y no experimental de este trabajo no permite realizar generalizaciones. Por último, no disponemos de información suficiente sobre la experiencia escritora previa del alumnado en la escuela y fuera de ella, lo que nos habría permitido interpretar de manera más completa las diferencias observadas entre las parejas. Así pues, para futuras investigaciones, sería interesante diseñar estudios que permitan comparar la planificación que realiza el alumnado en parejas con la realizada individualmente, así como incorporar instrumentos de investigación mediante los que extraer información sobre la experiencia escritora previa del alumnado.

Para concluir, consideramos que esta investigación aporta información valiosa para avanzar en una comprensión más profunda del proceso de planificación en la escritura infantil y orientar el diseño de intervenciones didácticas que favorezcan la enseñanza-aprendizaje de esta competencia en las aulas de primaria.

\section{Contribución de los autores}

Las dos autoras participaron en el diseño de la investigación, en la recogida de datos y en el análisis. La redacción del artículo se ha realizado de manera colaborativa. Mónica Aznárez-Mauleón ha liderado la redacción de las secciones 1, 2, 4.1, 5 y 7, y Maite López-Flamarique la de las secciones 3, 4.2 y 6.

\section{Referencias}

ARROYO, R.; SALVADOR, F. El proceso de planificación en la composición escrita de alumnos de la Educación Primaria. Revista de Educación, Madrid, n. 336, p. 353-376, 2005.

BEREITER, C.; SCARDAMALIA, M. The Psychology of Written Composition. Hillsdale: Lawrence Erlbaum Associates, 1987. 
BERNINGER, V. W.; SWANSON, H. L. Modifying Hayes and Flower's Model of Skilled Writing to Explain Beginning and Developing Writing. In: BUTTERFIELD, E. (ed.). Children's Writing: Toward a Process Theory of Development of Skilled Writing. Greenwich, CT: JAI Press, 1994. p. 57-81.

BODGAN, R. C.; BIKLEN, S. K. Qualitative Research for Education. 5. ed. Boston: Pearson, 2007.BURTIS, P. J.; BEREITER, C.; SCARDAMALIA, M.; TETROE, J. The Development of Planning in Writing. In: KROLL, B.; WELLS, C. G. (ed.). Explorations in the Development of Writing. Chichester: Wiley, 1983. p. 153-174.

CAMPS, A.; GUASCH, O.; MILIAN, M.; RIBAS, T. Actividad metalingüística: la relación entre escritura y aprendizaje de la escritura. In: CAMPS, A.; MILIAN, M. (ed.). Elpapel de la actividad metalingüistica en el aprendizaje de la escritura. Rosario: Homo Sapiens, 2000. p. 135-162.

DAIUTE, C. Do 1 and 1 Make 2? Patterns of Influence by Collaborative Authors. Written Communication, New York, v. 3, n. 3, p. 382-408, 1986. DOI: https://doi. org/10.1177/0741088386003003006

FLOWER, L.; HAYES, J. R. A Cognitive Process Theory of Writing. College Composition and Communication, Champaign, v. 32, n. 4, p. 365-387, 1981. DOI: https://doi.org/10.2307/356600

GALLEGO ORTEGA, J. L. La planificación de la expresión escrita por alumnos de educación primaria. Bordón, Madrid, v. 60, n. 2, p. 63-76, 2008.

GRAHAM, S.; MCKEOWN, D.; KIUHARA, S.; HARRIS, K. R. A Meta-Analysis of Writing Instruction for Students in the Elementary Grades. Journal of Educational Psychology, Washington, v. 104, p. 879-896, 2012. DOI: https://doi.org/10.1037/ a0029185.

GUTIÉRREZ, X. What Does Metalinguistic Activity in Learners' Interaction During a Collaborative L2 Writing Task Look Like? The Modern Language Journal, Hoboken, v. 92, n. 4, p. 519-537, 2008. DOI: https://doi.org/10.1111/j.15404781.2008.00785.x

KELLOGG, R. T. Attentional Overload and Writing Performance: Effects of Rough Draft and Outline Strategies. Journal of Experimental Psychology, Washington, v. 14, p. 355-365, 1988. DOI: https://doi.org/10.1037/0278-7393.14.2.355.

KRIPPENDORF, K. Metodología de análisis de contenido. Barcelona: Paidós, 1990.

LANGER, J. A. Children Reading and Writing: Structures and Strategies. Norwood: Ablex, 1986. 
LEE, K.; KARMILOFF-SMITH, A.; CAMERON, C. A.; DODSWORTH, P. Notational Adaptation in Children. Canadian Journal of Bebavioural Science, Ottawa, v. 30, n. 3, p. 159-171, 1998. DOI: https://doi.org/10.1037/h0087059

LIMPO, T.; ALVES, R. A.; FIDALGO, R. Children's High-Level Writing Skills: Development of Planning and Revising and Their Contribution to Writing Quality. British Journal of Educational Psychology, Chichester, v. 84, n. 2, p. 177-193, 2014. DOI: https://doi.org/10.1111/bjep.12020.

LITTLETON, E. B. Emerging Cognitive Skills for Writing: Sensitivity to Audience Presence in Five Through Nine-Year-Olds' Speech. Cognition and Instruction, Oxfordshire, v. 16, n. 4, p. 399-430, 1998. DOI: https://doi.org/10.1207/ s1532690xci1604_2

LÓPEZ, P.; TORRANCE, M.; RIJLAARSDAM, G.; FIDALGO, R. Effects of Direct Instruction and Strategy Modeling on Upper-Primary Students' Writing Development. Frontiers in Psychology, Lausanne, v. 8, p. 1-10, 2017. DOI: https:// doi.org/10.3389/fpsyg.2017.01054.

MACARTHUR, C.; HARRIS, K.; GRAHAM, S. Helping Students with Learning Disabilities Plan Compositions: Instruction in Cognitive and Metacognitive Strategies. In: BUTTERFIELD, E. (ed.). Children's Writing: Toward a Process Theory of Development of Skilled Writing. Greenwich, CT: JAI Press, 1994. p. 179-198.

MARLKLE, D. T.; WEST, R. E.; RICH, P. J. Beyond Transcription: Technology, Change, and Refinement of Method. Forum Qualitative Social Research, Berlin, v. 12, n. 3, p. 1-21, 2011. DOI: https://doi.org/10.17169/fqs-12.3.1564.

MATSUHASHI, A. Pausing and Planning: The Tempo of Written Discourse Production. Research in the Teaching of English, Urbana, v. 15, p. 113-134, 1981.

MCCUTCHEN, D. "Functional Automaticity” in Children's Writing: A Problem of Metacognitive Control. Written Communication, New York, v. 5, n. 3, p. 306-324, 1988. DOI: https://doi.org/10.1177/0741088388005003003

ROLDÁN-PREGO, L.; GONZÁLEZ-SEIJAS, R. M. Intervención en la planificación de la escritura en primer curso de enseñanza primaria. Revista de Estudios e Investigación en Psicología y Educación, Logroño, v. 3, n. 1, p. 9-18, 2016. DOI: https://doi.org/10.17979/reipe.2016.3.1.1174

SCHILPEROORD, J. On the Cognitive Status of Pauses in Discourse Production. In: OLIVE, T.; LEVY, C. M. (ed.). Contemporary Tools and Techniques for Studying Writing. New York: Springer, 2001. p. 61-88. DOI: https://doi.org/10.1007/97894-010-0468-8_4S 
CIENTIFIC SOFTWARE DEVELOPMENT GmbH. Atlas.ti: Qualitative Data Analisys. Versão 8. Berlin: ATLAS.ti Scientific Software Development GmbH, 2017.

SÉNÉCHAL, M.; HILL, S.; MALETTE, M. Individual Differences in Grade 4 Children's Written Compositions: The Role of Online Planning and Revising, Oral Storytelling, and Reading for Pleasure. Cognitive Development, Amsterdam, v. 45, p. 92-104, 2018. DOI: https://doi.org/10.1016/j.cogdev.2017.12.004.

STORCH, N. Collaborative Writing: Product, Process, and Students' Reflections. Journal of Second Language Writing, Amsterdam, v. 14, n. 3, p. 153-173, 2005. DOI: https://doi.org/10.1016/j.jslw.2005.05.002

STORCH, N. Collaborative Writing in L2 Contexts: Processes, Outcomes, and Future Directions. Annual Review of Applied Linguistics, Cambridge, v. 31, p. 275-288, 2011. DOI: https://doi.org/10.1017/S0267190511000079

SWAIN, M.; LAPKIN, S. Problems in Output and the Cognitive Processes They Generate: A Step Towards Second Language Learning. Applied Linguistics, Oxford v. 16, p. 371-391, 1995. DOI: https://doi.org/10.1093/applin/16.3.371

TAYLOR, J. S.; BOGDAN, R. Introducción a los métodos cualitativos de investigación. Barcelona: Paidós, 1987.

TORRANCE, M.; FIDALGO, R.; GARCÍA, J. N. The Teachability and Effectiveness of Cognitive Self-Regulation in Sixth-Grade Writers. Learning and Instruction, Leuven, v. 17, n. 3, p. 265-285, 2007. DOI: https://doi.org/10.1016/j. learninstruc.2007.02.003.

VILLAS BOAS, I. F. Process Writing in a Product-Oriented Context: Challenges and Possibilities. Revista Brasileira de Lingüistica Aplicada, Belo Horizonte, v. 14, n. 2, p. 463-490, 2014. DOI: https://doi.org/10.1590/S1984-63982014005000011

VYGOTSKY, L. S. Thought and Language. Cambridge: MIT Press, 1986.

WHITAKER, D.; BERNINGER, V. W.; JOHNSTON, J.; SWANSON, H. L. Intraindividual Differences in Levels of Language in Intermediate Grade Writers: Implications for the Translating Process. Learning and Individual Differences, Amsterdam, v. 6, p. 107-130, 1994. DOI: https://doi.org/10.1016/10416080(94)90016-7.

Data de submissão: 25/09/2019. Data de aprovação: 18/05/2020 